\title{
COMMISSION x6 (PHYSICAL OBSERVATIONS OF THE PLANETS)
}

\author{
President: Prof. M. Maggini. \\ SECRETARY: Dr B. Lyot.
}

La Commission s'est réunie à deux reprises: le 4 août et le 9 août. Le Président rend hommage aux membres que la Commission a perdus depuis l'assemblée de Paris: MM. les Profs. P. Stroobant et W. H. Pickering.

Il fait savoir que la Commission s'occupera aussi des observations physiques et de la nomenclature lunaire qui seront traitées par une sous-commission, la Commission ${ }_{7} 7$ s'occupant désormais seulement du mouvement de la Lune.

Il demande si l'on doit maintenir la petite sous-commission formée pour l'étude de la nomenclature de Mars. On est d'avis de la supprimer, la nomenclature de Schiaparelli constituant une base suffisante pour toute observation, étant donné que l'étude de la planète doit se rapporter plus aux grandes taches qu'aux menus détails. Il invite M. Donitch à parler sur ses observations de Mercure et de Saturne.

M. Donitch dit qu'il a photographié Mercure devant la chromosphère au spectrohéliographe avec la radiation $\mathrm{H}_{\alpha}$ lors du passage devant le Soleil du II mai, I937; il n'a observé aucun point ou anneau lumineux autour de la planète.

M. Lyot a photographié Mercure devant la couronne de $\mathrm{I}^{\prime}$ à $\mathbf{2}^{\prime} 3^{\prime \prime}$ du bord du Soleil et il arrive aux mêmes conclusions.

M. Donitch expose avec plus de détails ses observations sur la disparition de l'anneau de Saturne dont il est question dans le "Draft Report". Il en suit une discussion sur la visibilité et la couleur de l'anneau à laquelle prennent partie MM. Donitch, Phillips et le Président.

A propos des observations visuelles, M. Phillips rappelle que même avec des instruments modestes on peut déterminer la position et le mouvement des taches d'une planète. Il soumet à l'approbation de la Commission la résolution no. I.

Le Président appelle l'attention de la Commission sur la prochaine opposition de Mars. Il fait remarquer que l'observation visuelle doit se concentrer surtout sur les taches principales, tandis que pour les détails à la limite de visibilité elle doit rechercher la manière suivant laquelle notre œil arrive à une représentation durable des aspects très variables présentés par eux.

M. Waterfield est du même avis et cite son rapport surl'opposition de I935 présenté à la B.A.A.

Le Président saisit l'occasion pour faire remarquer que désormais l'observation visuelle ne peut plus suffire; il y a tout intérêt à exécuter des recherches physiques sur les planètes. Il répète encore une fois qu'il faut vaincre l'indifférence générale dont la physique planétaire est l'objet. A présent nous connaissons mieux les atmosphères des étoiles les plus éloignées que les atmosphères des planètes de notre système. C'est pour cela qu'il tient à signaler les recherches de M. Wildt, dont il a été question dans le "Draft Report" et qui constituent un véritable progrès dans la connaissance de l'enveloppe des planètes.

M. Wildt estime que l'interprétation des phénomènes observés à la surface de Jupiter et de Saturne et les recherches sur l'état interne de ces planètes demandent la connaissance des propriétés des gaz simples à de hautes pressions et dans un domaine étendu de températures. Il pense que les données expérimentales que nous 
possédons sont tout à fait insuffisantes; en conséquence il propose la résolution no. 2 , qui est approuvée par la Commission.

Le "Draft Report" est adopté avec une modification proposée par Mlle Harwood.

\title{
Résolutions adoptées
}

I. La Commission recommande aux observateurs de Jupiter et de Saturne au télescope, de porter plus systématiquement leur attention sur l'étude des couleurs, des niveaux et des mouvements des taches de ces planètes.

2. La Commission exprime le vœu que les physiciens expérimentaux possédant l'équipement nécessaire entreprennent l'étude de l'équation d'état des gaz simples, en particulier l'hydrogène, l'hélium, le méthane et l'ammoniac jusqu'aux pressions les plus hautes que l'on puisse atteindre.

\section{COMMISSION I8 (LONGITUDES)}

\section{(COMMISSION INTERNATIONALE MIXTE DES LONGITUDES)}

\author{
President: Général G. Perrier. \\ Secretary: Dr A. LAMberT.
}

La Commission internationale mixte des Longitudes a tenu deux séances: la première le vendredi 5 août, à $9^{\mathrm{h}} 30^{\mathrm{m}}$, la deuxième commune avec l'une des séances de la Commission de l'Heure, le samedi 6 août, à $10^{\mathrm{h}} 30^{\mathrm{m}}$.

Ière séance. Vendredi, 5 août 1938.

M. A. Lambert donne lecture du Rapport présenté à la Commission.* L'impression du Mémoire d'ensemble, relatif aux travaux de l'Opération internationale des Longitudes de I933, est commencée. Soixante et onze stations ont transmis leurs observations au Bureau international de l'Heure qui les a discutées. La propagation des ondes a fait également l'objet d'une étude dont on apporte les conclusions.

L'introduction contenant l'historique et l'exposé des méthodes de réductions employées, d'une part, et la monographie relative à la station d'Adelaïde de l'autre, constituent les deux premiers fascicules imprimés de la publication, soumis pour examen à la Commission.

L'ordre du jour des travaux de la Commission est ainsi fixé:

I. Influence du mouvement du pôle sur la longitude d'un point. (Proposition de M. F. da Costa Lobo.)

2. Quelle est la meilleure manière de traiter la question de l'équation personnelle dans les observations de passage? (Proposition de M. H. M. Jeffers.)

3. Communication de MM. R. Jouaust et N. Stoyko sur Les phénomènes de propagation des ondes radioélectriques et leur influence sur les opérations de détermination de longitude.

4. Détermination précise de la longitude par observations de distances zénithales extra-méridiennes au théodolite, par la méthode de l'obturateur oculaire. (Proposition de M. J. de Graaff Hunter.)

$$
\text { * P. I2I. }
$$

